\title{
La capacidad crítica como competencia transversal: La evaluación como
}

\section{herramienta}

\section{Critical capacity as cross-comprehensive competition: Evaluation as a tool}

\author{
Laura Redondo, Tania Corrás, Mª José Vázquez y Francisca Fariña \\ Grupo de Investigación PS1. Universidad de Vigo
}

\begin{abstract}
Resumen
En el marco educativo actual se hace necesaria una reflexión sobre el proceso de enseñanza-aprendizaje y las variables mediadoras que intervienen en él. En este sentido, el rendimiento académico se ve mediatizado por variables sociales, contextuales y personales. El presente estudio mide variables psicológicas como la autoeficacia y el optimismo, así como las expectativas de rendimiento y la calificación obtenida. A la luz de los resultados se pone de manifiesto la necesidad de valernos de herramientas para mejorar los procesos de enseñanza-aprendizaje, así como para optimizar los recursos del mismo, la mejora del rendimiento y el desarrollo integral del alumnado.

Palabras clave: Rendimiento, evaluación, EEES, Pensamiento crítico.
\end{abstract}

\begin{abstract}
In the current educational framework, it is necessary to reflect on the teaching-learning process and the mediating variables that intervene in it. In this sense, academic performance is mediated by social, contextual and personal variables. The present study measures psychological variables such as self-efficacy and optimism, as well as performance expectations and the qualification obtained. The results show the need to avail ourselves of tools to improve the teaching-learning processes, as well as to optimize the resources to improve the performance and the integral development of the students

Keywords performance, evaluation, EEES, Critical thinking.
\end{abstract}

\section{Introducción}

La era de la información demanda que las personas tengan que discernir entre los múltiples estímulos a los que se ven sometidas, obligándoles a tener que construir su propio juicio o punto de vista a partir de múltiples perspectivas (Paz, Molina y Sánchez, 2010). Esto hace necesario que el individuo adiestre su pensamiento egocéntrico o egoísta, pues éste lleva a no considerar losderechos y necesidades de los demás, ni apreciar el punto de vista de otros o las limitaciones del suyo propio (Paul y Edler, 2003). En este sentido, losprofesionales de la educación, así como asociaciones internacionalesy cierta legislación en materia educativa, recogen entre las finalidades y principiosdel sistema educativo, en todas sus etapas, el desarrollo del pensamiento (Chacón y Chacón, 2014; Ley Orgánica de Educación, 2006; UNESCO, 1995, 1998, 2009). Por su parte, el Consejo de Europa (2010) señaló, como uno de los objetivos fundamentales, reforzar la capacidad de acción del alumnado en la sociedad para defender y promover los derechos humanos, la democracia y el estado de derecho, más allá de la adquisición de conocimientos o competencias.

Sin embargo, a lo largo del tiempo diferentes investigaciones (McMillan,1987; Paz, Molina y Sánchez, 2010; Perkins, 1985; Whimbey, 1985), enfocadas a evaluar la enseñanza e implementación del sistema de pensamiento crítico encontraron que existe un pobre desarrollo del mismo en el alumnado. De esta manera, se ha ido evidenciando el fracaso del sistema educativo tradicional para desarrollar habilidades de pensamiento en la escuela, como Facione (1990) denunciaba, hace casi treinta años. A este respecto, Paul y Edler (2005) establecen que uno de los lastres de la educación ha sido que el profesorado asume que exponer un contenido implica, automáticamente, que el alumnado adquiere el procedimiento adecuado de cómo manejar dicho conocimiento de manera apropiada. Obviando, que para que esto se produzca el docente ha de realizar una labor de apoyo activo para que asimilen los contenidos de manera profunda y significativa. Proceder que no sólo se ha de seguir en los niveles educativos obligatorios, sino incluso en la Educación Superior. Puesto que en esta etapa también caben que existan carencias en el desarrollo del pensamiento. Así, en ella se deberían promover funciones psicológicas superiores, tales como la reflexión crítica y el razonamiento moral, las capacidades de comunicación o la sensibilización ante la diversidad, entre otras, siguiendo documentos de referencias educativa como los de la UNESCO (Loredo y Ferreira, 2011). Concretamente, tal y como se señaló en la Conferencia Mundial sobre la Educación Superior: La Educación Superior para el siglo XXI (UNESCO, 1998) es necesario plantear nuevos modelos pedagógicos para la transmisión y generación del conocimiento, formando a los estudiantes como ciudadanos bien informados y profundamente motivados, provistos de un sentido crítico y capaces de analizar los problemas de la sociedad, buscar soluciones y asumir responsabilidades. 
Por ende, el planteamiento de este nuevo marco educativo implica un cambio de roles del alumnado y del profesorado, donde se elimina la asimetría de poder en función de quien posee el conocimiento, pasando a una diferenciación en función de los niveles de competencia entre ambos (Paulino y Palmieri, 2009). Esta es, precisamente, la concepción que subyace al Espacio Europeo de Educación Superior (EEES). Donde, siguiendo el informe UNESCO, La educación encierra un tesoro, se establecen cuatro pilares que deberían sustentar la educación para el siglo XXI: Aprender a conocer, aprender a hacer, aprender a vivir juntos y aprender a ser (Delors, 2006); o sea, en los conocimientos, los procedimientos, la convivencia y el desarrollo personal. Así, como afirman diferentes autores (p.e., Ismarson y Vargas, 2010; Martí-Vilar, Vargas, Moncayo y Martí, 2014) se debe plantear también en la Enseñanza Superior competencias que otorguen importancia al desarrollo del pensamiento crítico, puesto que el universitario es un ente activo que puede juzgar la pertinencia de los conocimientos que adquiere para construir y dar sentido a sus valores, emociones $y$ aspiraciones.

En relación ala adquisición del pensamiento crítico, Díaz-Barriaga (2001) afirma que puede adquirirse a través de métodos diferentes, entre ellos el dialógico. Este asume que la ausencia de capacidad crítica conlleva al prejuicio, la parcialidad, y la irracionalidad (Paul, 1992; Walsh y Paul, 1998), y por tanto un mecanismo facilitador de injusticia. En relación con esto, se encuentra la capacidad de ser objetivos y de evaluar. Por ello, involucrar al alumnado en el proceso de evaluación supone una oportunidad de aprendizaje que desarrolla y potencia su pensamiento crítico (Klooster, 2001; Rodríguez et al., 2012; Sambell y McDowell, 1988; Sivan, 2000); además de otras múltiples competencias; entre ellas, el aprendizaje autónomo y la autoconfianza (Brew, 2003).Asimismo, a través de la participación activa en los procedimientos de evaluación, el alumnado puede regular sus propios mecanismos de aprendizaje (Boud, 2006; Boud, 2010; Nicol, 2009; Rodriguez et al., 2012). Esto requiere que exista capacidad crítica para llevarlo a acabo de forma fundamentada. En esta línea, dentro de la evaluación orientada al aprendizaje se puede emplear la evaluación por pares, la autoevaluación, y la coevaluación (Carless, Joughin y Mok, 2006, y Dochy, Segers y Sluijsmans, 1999).

Partiendo de lo anterior, el objetivo de este estudio erafacilitar el aprendizaje del alumnado. A la par,analizarsi el alumnado universitario utiliza un sistema de pensamiento crítico, en función de la evaluación que hace del trabajo propio y del ajeno. Concretamente, se consideró la parcialidad versus imparcialidad del alumnado para evaluar el trabajo de exposición de final de materia, del propio grupo (intragrupal) y de los otros grupos de clase (intergrupal).Para tal fin, como mecanismo de evaluación, se diseñó una rúbrica que el alumnado tenía que cumplimentar como mecanismo de evaluación. Se optó por la rúbrica por tratarse de un instrumento de medición de las competencias que ha resultado de gran utilidad dentro de la enseñanza superior (Novo, Seijo y Redondo, 2016).

\section{Método}

\section{Procedimiento}

Se facilitó al alumnado una rúbrica para que pudieran evaluar el desempeño de los grupos (intragrupal e intergrupal), en la exposición final de una materiade primer curso de la titulación de Ciencias de la Actividad Física y del Deporte. Todos los participantes lo hicieron de manera voluntaria, Se les informó que el profesorado no consideraría su valoración para establecer la calificación de los trabajos. Quienes participaron tenían que evaluar el trabajo de su equipo y el de otros 3 grupos con los que compartían horario.

\section{Participantes}

La muestra la conformaron un total de 104 estudiantes de primero de la titulación del grado de Ciencias de la Actividad Física y del Deporte, de la Universidad de Vigo

\section{Instrumentos}

Se diseñó una rúbrica compuesta por 8 ítems que valoran: a) el desempeño en la exposición (Claridad, Lenguaje Formal y Coordinación entre los miembros del grupo y Conexión); b) los contenidos de la misma (Uso de medios Audiovisuales, Trasferencia de los conocimientos a la práctica y Originalidad), y c) Puntuación global percibida. Cada ítem tenía que responderse en una escala continua de 1 a 10 , siguiendo la puntuación del propio expediente académico, en base a la Resolución del 15 de septiembre del 2011, de la Secretaría Xeral Técnica da Consellería de Educación e Ordenación Universitaria.

\section{Análisis de datos}

Para el análisis de datos se ha utilizado metodología descriptiva. Además, se ha aplicado la prueba estadística t de student para muestras relacionadas con el objetivo de explorar si se evidencian diferencias significativas entre las medias de las valoraciones intragrupal y la intergrupalen cada uno de los ítems que componen la rúbrica.

\section{Resultados}

Dentro del análisis estadístico, procedimos a cotejar la valoración de cada una de las categorías para proceder a continuación a la comparación de los resultados entre los distintos grupos previamente establecidos (Tabla 1). Si bien en el ítem Puntuación global percibida no se observan diferencias significativas entre el propio grupo y el resto de grupos, en las puntuaciones pormenorizadas se observan diferencias en la evaluación. Concretamente, dentro de la categoría de Desempeño, se observan diferencias estadísticamente significativas en las áreas evaluadas de Coordinación $(\mathrm{t}=2.13, \mathrm{p}=.039)$, Claridad $(\mathrm{t}=2.84, \mathrm{p}=0.006)$, Lenguaje formal $(\mathrm{t}=2.048, \mathrm{p}=0.044) \mathrm{y}$ Conexión $(\mathrm{t}=4.11 ; \mathrm{p} \leq .001)$. Dentro del contenido de la exposición los datos muestran diferencias significativas en la Trasferencia de los conocimientos a la práctica 
$(\mathrm{t}=4.22, \mathrm{p} \leq .001)$ y la Originalidad $(\mathrm{t}=4.26, \mathrm{p} \leq .001)$. En el área de Uso de medios Audiovisuales no se observan diferencias estadísticamente significativas, siendo la única área en la que no se encuentran.

Tabla 1.

Prueba $t$ de student para muestras relacionadas de las valoraciones intragrupalese intergrupales.

\begin{tabular}{|c|c|c|c|c|}
\hline & $\begin{array}{c}\mathrm{M} \\
\text { (D. T) }\end{array}$ & $\begin{array}{c}\text { M } \\
\text { (D. T.) }\end{array}$ & $t$ & $\mathrm{p}$ \\
\hline Puntuación Global & $\begin{array}{l}8.50 \\
(0.81)\end{array}$ & $\begin{array}{l}8.35 \\
(0.67)\end{array}$ & 0.75 & .457 \\
\hline Coordinación & $\begin{array}{l}9.07 \\
(1.85)\end{array}$ & $\begin{array}{l}8.47 \\
(1.20)\end{array}$ & 2.13 & .039 \\
\hline Claridad & $\begin{array}{l}8.27 \\
(0.92)\end{array}$ & $\begin{array}{l}7.84 \\
(1.23)\end{array}$ & 2.84 & .006 \\
\hline Lenguaje Formal & $\begin{array}{l}8.07 \\
(1.04)\end{array}$ & $\begin{array}{l}7.79 \\
(1.22)\end{array}$ & 2.05 & .044 \\
\hline Coordinación & $\begin{array}{l}8.61 \\
(1.38)\end{array}$ & $\begin{array}{l}7.60 \\
(1.78)\end{array}$ & 4.11 & .001 \\
\hline $\begin{array}{l}\text { Uso de medios } \\
\text { audiovisuales }\end{array}$ & $\begin{array}{l}8.24 \\
(1.07)\end{array}$ & $\begin{array}{l}7.88 \\
(1.35)\end{array}$ & 1.92 & .058 \\
\hline $\begin{array}{l}\text { Trasferencia a la } \\
\text { práctica }\end{array}$ & $\begin{array}{l}8.77 \\
(0.98)\end{array}$ & $\begin{array}{l}8.09 \\
(1.15)\end{array}$ & 4.22 & .001 \\
\hline Originalidad & $\begin{array}{l}8.47 \\
(1.10)\end{array}$ & $\begin{array}{l}7.73 \\
(1.19)\end{array}$ & 4.26 & .001 \\
\hline
\end{tabular}

Nota: $\mathrm{M}=$ Media, $\mathrm{DT}=$ Desviación Típica, $\mathrm{t}=$ estadístico $\mathrm{t}$ de student para muestras relacionadas, $\mathrm{p}=$ significación estadística al $95 \%$

En suma, en la puntuación global, siendo esta la impresión subjetiva del desempeño de la exposición, no se observan diferencias, resultando estas calificaciones con mayor puntuación y menor variabilidad. Por otro lado, en el análisis particular de cada una de las áreas se observa que la percepción del desarrollo de las competencias se realiza de manera distinta, con una tendencia a valorar mejor al propio grupo en6 de las 7. Justamente, es en el Uso de medios audiovisuales en la que no se observan diferencias, siendo la más instrumental de todas ellas. A nivel descriptivo, los resultados muestran una tendencia, en todas las áreas, a la valoración del propio grupo de manera significativamente mayor (ver Figura 1).

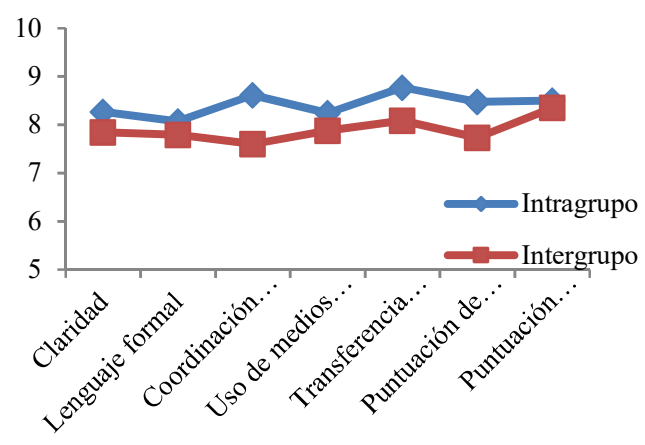

Figura 1. Diferencias entre las evaluaciones intra e intergrupales.

\section{Conclusiones y discusión}

Los resultados encontrados ponen de manifiesto que los estudiantes universitarios de primer curso, que participaron en este estudio, llevan a cabo la evaluación del desempeño propio y ajeno de manera parcial, tendiendo a puntuar mejor la propia ejecución. Lo que evidencia la falta de un pleno desarrollo de la capacidad crítica, pese a que todo universitario debe contar con esta competencia. Por su parte, Paz, Molina y Sánchez (2010) también encontraron que la mayoría del alumnado de Educación Secundaria Obligatoria no ejecutaba el pensamiento crítico.

De esta manera, se evidencia la necesidad de potenciar especialmente estas capacidades en las enseñanzas previas al ingreso en la Educación Superior. Igualmente, se muestra relevante entrenarlas durante la formación universitaria, para lograr que el estilo de procesamiento crítico tenga mayor saliencia cognitiva y coductual. Esto, conllevaría a que los egresados no sólo tengan competencias de corte profesional, sino también de corte moral, para que resulten ciudadanos eficaces, capaces de razonar éticamente y actuar en beneficio del bien público (Paul y Elder, 2005). Sobre todo teniendo en cuenta que el pensamiento crítico incentiva la participación, la responsabilidad con las ideas propias, pero también involucra el intercambio y la tolerancia (Chacón y Chacón, 2014), en la línea del planteamiento del EEES (Delors, 1996).

Por otra parte, cabe utilizar la evaluación del trabajo propio y ajeno, a través de una rúbrica, como una vía de potenciación de la capacidad crítica en el ámbito universitario. A la vez que se enseñan los mecanismos de aprendizaje y se estimula el interés por el desarrollo de las competencias (McDonald, Boud, Francis y Gonczi, 2009), lo que genera expectativas que inciden en la movilización de los recursos personales del alumnado (Redondo, Alonso, Fariña, Cuevas, y Acuña, 2017). Sin duda, para que esto sea factible es necesario también formar y motivar al profesorado para que lo considere en su guía docente; acorde con la UNESCO (1998), cuando afirmaba que el docente ha de ser elcatalizador para el alumnado efectúe eficazmente el análisis creativo y crítico

\section{Referencias}

Arce, R. Novo, M., Seijo, D., y Redondo, L. (2016). Evaluación de las competencias en el trabajo fin de máster. En P. Membiela, N. Casado, y M. I. Cebreiros (Eds.), La docencia universitaria: Desafios y perspectivas (pp. 215-219). Ourense: Educación Editora. ISBN: 978-84-15524-30-4

Boud, D. (2006). Foreword. En C. Bryan y K. Clegg (Eds.), Innovative Assessment in Higher Education (pp. 17-19). New York: Routledge.

Boud, D. (2010). Assessment 2020: Seven propositions for assessment reform in higher education. Sydney: ALTC.

https://www.uts.edu.au/sites/default/files/Assessment2020 propositions final.pdf

Brew, A. (2003). La autoevaluación y la evaluación por los compañeros. En S. Brown y A. Glasner (Eds.), Evaluar en la Universidad. Problemas y nuevos enfoques (pp. 179-189). Madrid: Narcea.

Carless, D., Joughin, G., y Mok, M. (2006). Learning-oriented assessment: principles and practice. Assessment and evaluation in Higher Education, 
$31(4)$,

395-398.

http://dx.doi.org/10.1080/02602930600679043

Chacón, M.A., y Chacón. C.M. (2014). La lectura crítica: Herramienta para la promoción del pensamiento crítico en la formación inicial docente. Legenda, 18, 99-123.

Consejo de Europa (2010). Carta del Consejo de Europa sobre la educación para la ciudadanía democrática y la educación en derechos humanos. Instrumentos jurídicos, Recomendación CM/Rec (2010)7.

Delors, J. (1996). La educación encierra un tesoro. Madrid: Santilla- Ediciones UNESCO. http://www.unesco.org/education/pdf/DELORS_S.PD $\mathrm{F}$

Díaz-Barriga, F. (2001). Habilidades de pensamiento crítico sobre contenidos históricos en alumnos de bachillerato. Revista Mexicana de Investigación Educativa, 6(13), 525-554 http://www.redalyc.org/articulo.oa?id=14001308\&iC veNum $=172$

Dochy, F., Segers, M. y Sluijsmans, D. (1999). The Use of Self-, Peer and Co-Assessment in Higher Education: a Review. Studies in Higher Education, 24 (3), 331-350.

http://dx.doi.org/10.1080/03075079912331379935

Facione P. (1990) Critical thinking: a stament of expert consensus for purposes of educational assessment and instruction. CA: California State University, Fullerton.

Ismarson, I.y Vargas, O. (2010). Resisting through Corporate Values.Tesis Doctoral, School Of Economics And Management, Lund University . http://lup.lub.lu.se/luur/download?func=downloadFile \&recordOId $=1615995 \&$ fileOId $=2435875$

Klooster, D. (2001). What is Critical Thinking? Thinking Classroom. A Journal of Reading, Writing and Critical Reflection, 4, 36-40.

Ley Orgánica 2/2006, de 3 de mayo, de Educación. (2006).

https://www.boe.es/buscar/pdf/2006/BOE-A-2006-78 99-consolidado.pdf

Loredo, J. C. y Ferreira, A.A.L (2011). Aventuras y desventuras de la educación en el Reino de Psicolandia: el supuesto respaldo científico del Espacio Europeo de Educación Superior. Athenea Digital, Noviembre, 79-97. http://atheneadigital.net/article/viewFile/v11-n3-lored o-ferreira/858-pdf-es

Martí-Vilar, M., Vargas, O.H., Moncayo, J.E., y Martí, J.J. (2014). La formación en razonamiento moral y pensamiento crítico en la Educación Superior Brazilian Geographical Journal: Geosciences And Humanities Research Médium, 5(2), 398-414. https://www.researchgate.net/profile/Manuel_Marti Vilar/publication/268506888_La formacion_en_razo namiento_moral_y_pensamiento_critico_en_la_Educa cion_Superior/links/546df4c00cf $29806 \mathrm{ec} 2 \mathrm{e} \overline{6} 28 \mathrm{f} / \mathrm{La}-\mathrm{f}$ ormacion-en-razonamiento-moral-y-pensamiento-criti co-en-la-Educacion-Superior.pdf

Mc Donald, R., Boud, D., Francis, J., y Gonczi, A. (2009). Nuevas perspectivas sobre la evaluación. OrganizaciónInternacional del Trabajo.

McMillan, J.H. (1987). Enhacing college students'critical thinking: A review of studies.
Research in Higher Education, 26, 3-29. http://www.jstor.org/stable/40195772

Nicol, D. (2009). Transforming Assessment and Feedback: Enhancing integration and empowerment in the first year. Mansfield: EnhacementThemes.

Paul, R (1990). Critical and reflective thinking: a philosophical perspective. Jones y Idol (Eds.), Dimensions of thinking and cognitive instruction. Hillsdale: Erlbaum

Paul, R. y Elder L. (2005) Una guía para los educadores en los estándares de competencia para el pensamiento crítico. Fundación para el pensamiento crítico.https://www.criticalthinking.org/resources/PD F/SP-Comp Standards.pdf

Paul, R., y Elder, L. (2003). La mini- guía para el pensamiento crítico. Conceptos y herramientas. California: Fundación para el pensamiento crítico.

Paulino, L. R. P. y Palmieri, M. W: A. R. (2009) "A história da psicologia social contada da perspectiva do aluno que nãofez a 'tarefa de sempre'. Athenea digital, 17 163-178. http://www.raco.cat/index.php/Athenea/article/view/1 80702/233232

Paz, J.S., Molina, E.C., y Sánchez, L.P. (2010). Pensamiento crítico y capacidad intelectual. Faisca: Revista De Altas Capacidades, 15(17), 92-110. http://revistas.ucm.es/index.php/FAIS/article/view/85 05

Perkins, D.N. (1985). Postprimary education has a little impact on informal reasoning. Journal of Educational Psychology, 77, 562-570.

Redondo, L., Alonso, A., Fariña, F., Cuevas, M., y Acuña, A. (junio, 2017). Expectativas de rendemento e aprendizaxe: $O$ peso do optimismo. $\mathrm{V}$ Conferencia Internacional e Nacional de Docencia Universitaria (CINDU). Vigo, Pontevedra.

Rodríguez-Gómez, G., Ibarra-Sáiz, M. S., Gallego-Noche, B., Gómez-Ruiz, M. Á., y Serra, V. Q. (2012). La voz del estudiante en la evaluación del aprendizaje: un camino por recorrer en la universidad. RELIEVE. Revista Electrónica de Investigación y Evaluación Educativa, 18(2), 1-21. http://dx.doi.org/10.7203/relieve.18.2.1985

Sambell, K. y McDowell, L. (1998). The construction of the hidden curriculum: messages and meanings in the assessment of student learning. Assessment and Evaluation in Higher Education, 23, 391-402. http://dx.doi.org/10.1080/0260293980230406

Sivan, A. (2000). The implementation of peer assessment: an action research approach. Assessment in Education, 7(2), 193-213. http://dx.doi.org/10.1080/713613328

UNESCO (1995) Documento de política para el cambio y el desarrollo en la educación superior. París: Unesco.http://unesdoc.unesco.org/images/0009/00098 9/098992s.pdf

UNESCO (1998) Conferencia mundial sobre la Educación Superior La Educación Superior para el siglo XXI: Visión y Acción. Madrid: Ediciones UNESCO.http://unesdoc.unesco.org/images/0011/001 163/116345s.pdf 
UNESCO. (2009). Comunicado Conferencia Mundial sobre la Educación Superior: Las nuevas dinámicas de la educación superior y la investigación para el cambio social y el desarrollo. Paris: UNESCO. http://www.scielo.org.mx/pdf/peredu/v31n126/v31n1 26a8.pdf

Walsh, D., y Paul, R. (1998). The goal of critical thinking: From educational ideal to educational reality. Washington, DC, American Federation of Teachers.

Whimbey, A. (1985). Test results from teaching thinking, en Costa A. L. (Ed.), Developing minds: $A$ resource book for teaching thinking (pp. 269-271).

\section{Agradecimientos}

Este trabajo se enmarca dentro del Contrato Programa 2017 de la Universidad de Vigo 